\title{
Investigations of the key mechanism of Carbon-Nanotube Actuators and their dependencies
}

\author{
Sebastian Geier, Johannes Riemenschneider, Thorsten Mahrholz, \\ Peter Wierach, Michael Sinapius; \\ Institute of Composite Structures and Adaptive Systems, \\ DLR e.V. (German Aerospace Center), \\ Lilienthalplatz 7, Braunschweig, Germany
}

\begin{abstract}
Future adaptable applications require electro-mechanical actuators with a high weight-related energy. Among modern multi-functional materials carbon nanotubes (CNTs) have some special characteristics which give them the potential to solve this demand. On the one hand raw CNTs have excellent mechanical properties like their low density $\left(1330 \mathrm{~kg} / \mathrm{m}^{3}\right)$ and very high estimated stiffness of about $1 \mathrm{TPa}$. On the other hand CNTs have the ability under presence of ions, wired like a capacitor and activated by a charge injection to perform a dimension-change (length of $\mathrm{C}-\mathrm{C}$ bondings). Calculations and experiments present achievable active strains of $1 \%$ at low voltage of $\pm 1 \mathrm{~V}$ what qualifies CNT-based materials for leightweight powerful actuators.
\end{abstract}

In this paper the former work done with actuators using CNT-containing mats and Nafion as solid electrolyte is evaluated by analyzing the two main-components in more detail. On the one hand the CNT-based model-material SWCNT-mats called Bucky-paper (BP) and on the other hand ion donating electrolytes in liquid-phase like a $\mathrm{NaCl}$-solution and its solid equivalent Nafion as thin-foils are tested. Additional methods of fabrication, preparation and characterization of the CNT-powder and the manufactured BPs containing randomly oriented single-walled carbon nanotubes (SWCNTs) are presented which provide a deeper system-understanding. Both materials (BPs and Nafion-foils) are intensively investigated in different deflection-test-rigs due to their structural assembly. This paper presents a method for electro-mechanical measurements of BPs in an in-plain test set-up which avoids sensing secondary effects like thermal expansion or mass-transport and confirm that BP-deflection should only be a capacitydriven effect. Nafion as solid electrolyte will be tested in an out-of-plane facility to measure its possible actuation within the lamellar-direction. With this approach the dependencies of each component and their individual characters on the deflection can be estimated. The active response can be referred to the internal structure of both components as well as of the whole structural assembly.

The results give a certain direction to a BP-optimization referring to active strain, density, structural integrity and conductibility. In addition to these facts the active character of BPs using CNTs of different suppliers and Nafion is analyzed. These investigations are of particular importance for detection of global dependencies and using both materials in a hybrid-assembly like solid actuators which are needed for structural applications.

Keywords: Carbon nanotube, actuator, Bucky paper, liquid electrolyte, solid electrolyte

\section{INTRODUCTION}

Among multi-functional materials that convert electrical into mechanical energy there are three groups of actuators which are well researched and almost commercially available (see fig. 1).

The most popular active material are piezoceramics (PZT see fig. 1) which contract/expand within an electric field. This kind of actuator is used in most of the realized adaptable applications. With voltages up to $1500 \mathrm{~V}$ a maximum strain of $0,15 \%$ can be reached. Beside the stiffness these properties are too low for structural applications and often uneconomical. The second interesting kind of material are Shape-Memory-Alloys (SMA) which change crystal configuration with temperature. On the one hand

Further Author Information: Send Correspondence to Sebastian Geier

E-mail: Sebastian.Geier@dlr.de, Telephone: +495312952355 


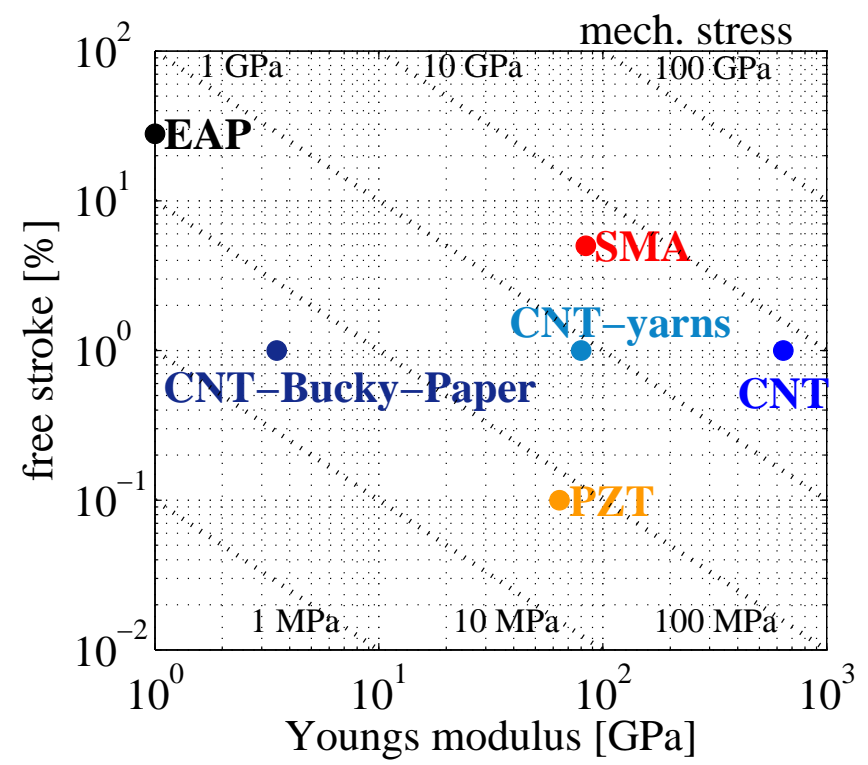

Figure 1. comparison of different modern actuator-systems ${ }^{1}$

free strains of $8 \%$ are possible but on the other hand it shows slow reaction, time-depentend deflection is unkown and the long-term behavior is unstable. The third relevant group are electro-active-polymers which are the topic of many present research-groups and are near by maturity. The chemical or magnetical activated process causes a volume-change which can generate deflections of $200 \%$ within high frequencies (depending on mechanism $>40 \mathrm{~Hz}$ ). Because of the elastic polymer-matrix there is nearly no stiffness and therefore the system is not suitable for structural applications. Previous works demonstrate that carbon nanotubes can generate high forces at very low voltages ${ }^{2}$ and with consideration of their mentioned material-characteristics ${ }^{3,4}$ it might be the basis of future lightweight actuators for adaptable applications.

\section{STATE OF THE ART}

In order to implement a CNT-based actuator in structural applications it is essential to incorporate a solid durable ionic electrolyte. A few publications examine the use of solid electrolytes like Nafion or Fumion. ${ }^{5-8}$ The described actuators are build up of BPs coated on one or both sides with solid electrolytes. The active deflection-measurements were often made by using bimorph-benders. This kind of set-ups shows on the one side high deflection-rates but implies on the other side the risk of measuring secondary expansion-effects like mass transfer induced by an electric field along the field lines ${ }^{9,10}$ or thermal caused volume-changes. Therefore if the actuator-set-up is unsymmetrical with one electrolytelayer and for eliminating the mentioned secondary-effects the test should be done in an out-of-plane configurated test-rig ${ }^{11}$.

Extensive prework of this paper investigating CNT-Nafion hybrid actuators depicted capacitive character but the whole performance was neither reproducible nor comparable to the results of BPs acting in liquid electrolytes (see fig 2).

Within the great amount of publication on ionic electrolytes and their use as bending actuators like artificial muscles there are also investigations about the deflection of solid electrolytes-layers in electric fields using the out-of-plane set-up. ${ }^{12}$ With this background the unstable results taken from the out-ofplane measurement cannot be only related to the CNT-BP-response. There is the need to investigate what individual active-characteristic thin polymer-layers could have like they are used in hybrid solid actuators. It is essential to get an idea of the global system-response which seems to consist of an electrolyte- and a BP-fraction.

Another source of influence on the deflection of CNT-based actuators might be the mechanical and structural characteristics of the BP itself. Publications ${ }^{8}$ gave an idea about the dependencies between the Young's modulus and the deflection of a Bucky-Paper. Because of these relations it is useful to do more detailed investigations on the BP-preparation-process referring to deflection-tests in a definite test 

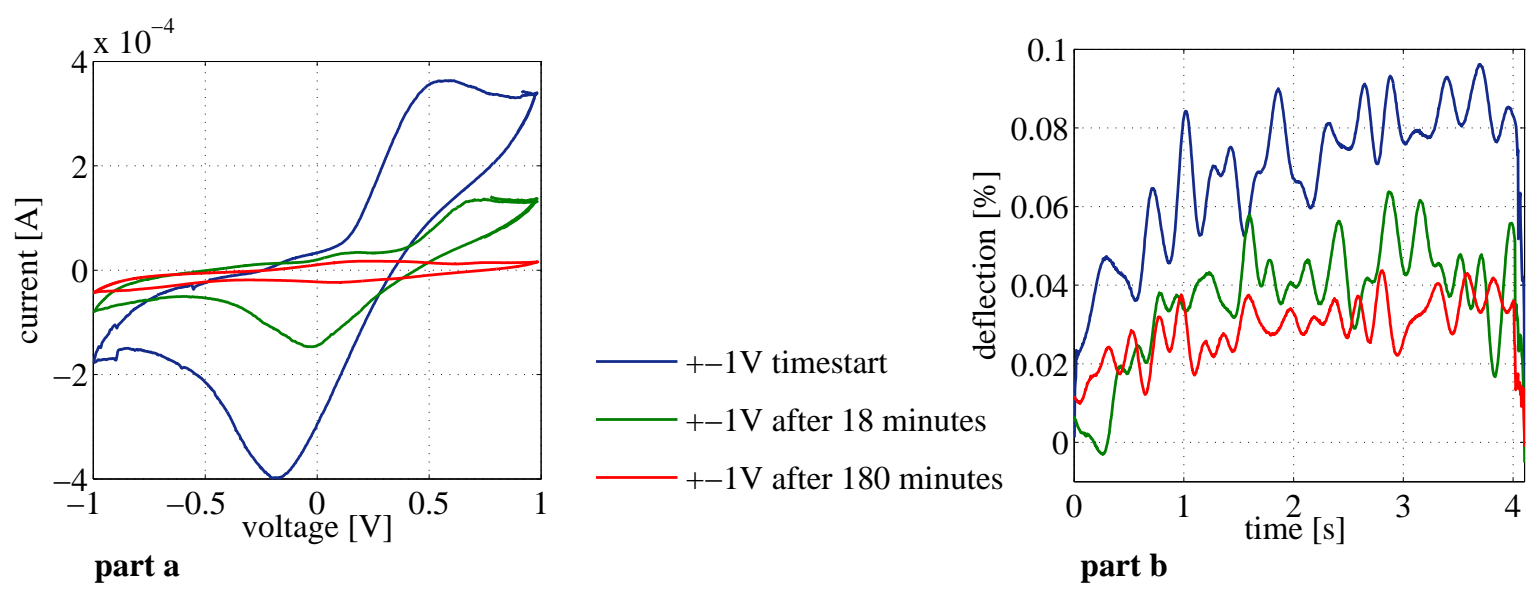

Figure 2. part a: cyclovoltametric curves for $\pm 1 \mathrm{~V}$ at different times with a sweeprate of $80 \mathrm{mV} / \mathrm{s}$, part b: measured displacements at the same times

method like it is the in-plane set-up. This kind of facility is well known, ${ }^{1,13-15}$ avoiding secondary-effects and the results are checked by theoretic models. ${ }^{16,17}$

This approach will guide to a better understanding of the individual components and the dependencies of their mechanical, structural and electrical characteristics on the global system-response.

\section{PREPARATION}

The optimization of several BP-characteristics like stiffness, durability for processing, low shrinking and high conductibility for its application as actuator is the aim of the described preparation-process. Because of their single walls and therefore 10-times higher surfaces-area single-walled carbon nanotubes (SWCNTs) are chosen for basis-material. The SWCNTs should not be surface-modified otherwise the free $\pi$-electrons will be fixed by side-groups which is no standard for conductible applications - like actuators.

As summarized in table 1 a broad range of SWCNT-raw material is used to study the influences of purity and processability on BP-quality .

All Bucky-papers are fabricated on the same way like it is shown in figure 3 and described in the following chapter. The only variation takes place after the second process step when three CNT-solutions are centrifuged at $4000 \mathrm{~g}$ for five, ten or fifthteen minutes.

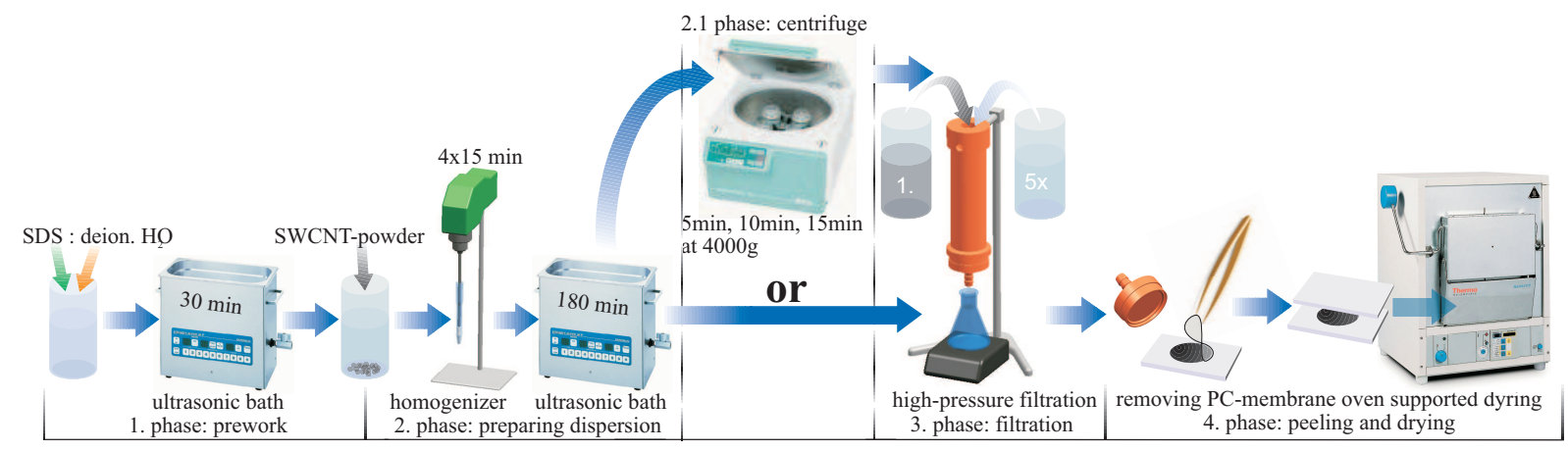

Figure 3. process-steps of Bucky-paper fabrication

This manufacturing-process was adapted and extended from one ${ }^{18}$ of many publications. In the prework-step the solution of $1 \mathrm{wt} \%$ of the surfactant sodium dodecyl sulfate (SDS) is prepared in deionized water by using 30 minutes an ultrasonic bath. In the next step the $0,1 \%$ of SWCNT-powder is put into $5 \mathrm{ml}$ of the SDS-dispersion and homogenized for one hour in a manual homogenizer. In the second part of this phase a three hour long ultrasonic bath takes place after that the CNT-dispersion can be centrifuged or directly filtrated. Out of one individual CNT-raw material lot four dispersions have to be set up so that all process-varations can be mapped. Usually $60 \%$ of the CNT-material will be sedimented after the 
centrifuge. The third process-phase includes the high-pressure filtration at 6 bar and three to five-times of $\mathrm{BP}$-washing under pressure with deionized water to remove the surfactant. The fourth and final process step begins with peeling the thin polycarbonate-membrane off the CNT-mat. As preparation for a flat Bucky-paper later on the wet CNT-mat is layed up between two teflon-plates. This set-up will be heated in an oven to $80^{\circ} \mathrm{C}$ for several hours to dry the BPs.

The produced papers are cut into small stripes of about $20 \mathrm{~mm} \times 4 \mathrm{~mm}$ in order to simplify the analysis of the samples within the special facilities. The specimen are tested for conductibility with a four-point test-method. Their internal structure will be optical investigated by a scanning electron microscope (SEM). Within this investigations the BP-surfaces and by a cyrogen-cut the internal, unaffected structure is examined. The Young's modulus will be measured via a dynamic-mechanical analysis at a frequency of $1 \mathrm{~Hz}$ for 20 minutes. Their active strain is tested in an in-plain optical measurement-facility. The bulk-density of the Bucky-paper is calculated by the measured volume and weight. Finally the well characterized Bucky-papers allow in comparison to each other BP - every one is made of different raw materials and in different ways - a statement of the favorable material and process-steps.

The common solid electrolyte Nafion which was used for the hybrid actuators will be used in this paper as single layers for investigations of its own active behavior. The layers are fabricated out of a solution by using of two parts of the purchasable DuPont Nafion solution and one part of dimethylformamide (DMF) for better processability. This dispersion has to be treated by a magnetic stirrer due to a better homogeneity for ten minutes. After that process the Nafion solution can be spreaded by a brush on a Teflon(PTFE)-plate. After 20 minutes the first layer is not totally dried but dimensionally stable, so that the second layer can be put on it. Afterwards the same procedure is repeated in cycles until the stack reaches the desired thickness or layer number. Experiments reveal that just a monolayer of Nafion is difficult to handle especially when it has to be removed off the Teflon-plate by a scalpel. It is found that two layers are a minimum for good processability. As final step the Nafion-sheet has to be coated with a thin gold foil for better conductivity to the copper electrodes and for better optical signals on its surface.

\section{EXPERIMENTAL SET-UP}

As already stated the different types of Bucky-papers are analyzed in an in-plane-test set-up. Figure 4 demonstrates the assembly of this facility. With this test-rig it is possible to measure optical deflections of Bucky-papers within their propagation direction while they are electrical activated.

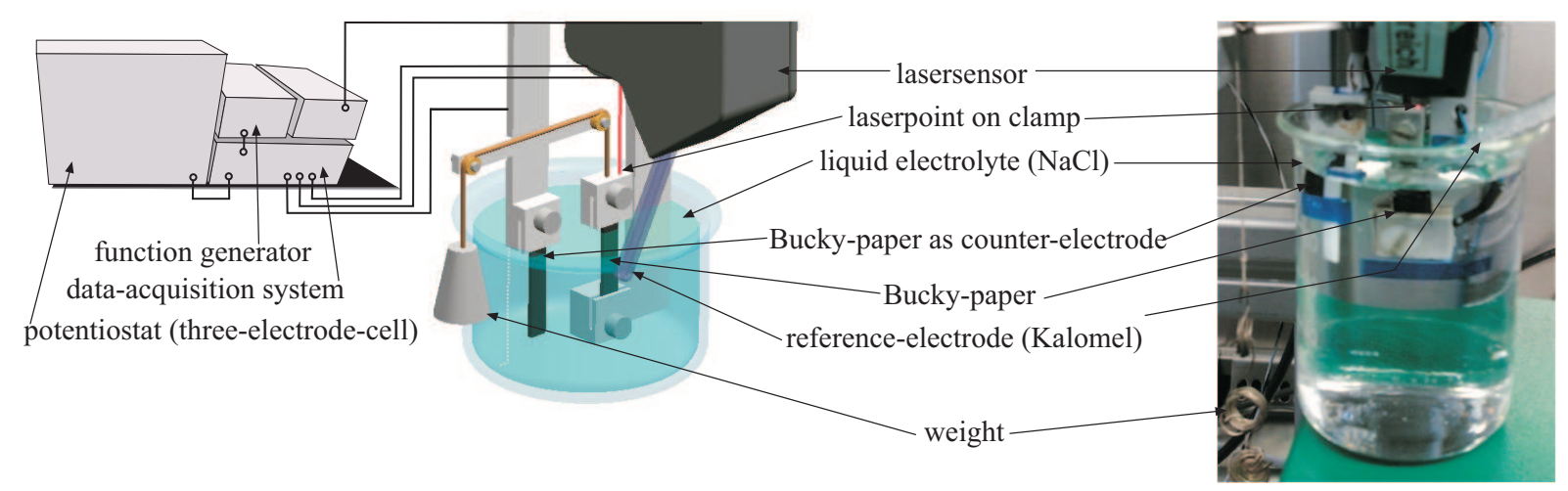

Figure 4. test set-up for measuring the active strain of different Bucky-paper lots

The analyzed Bucky-Paper is fixed at its ends with clamps. The bottom-clamp consists of a platinumelectrode for electrical activation. It is fixed to the rest of the test-rig. The top-clamp is movable freely. Tightened by a wire with a fixed weight at the end the Bucky-Paper shift can be measured by a laserdistance-device (Micro-Epsilon optoNCDT LD 1607-0,25) which is focused on the white coated surface of the top-clamp. The whole Bucky-paper is plunged into an aqueous monomolare sodium chloride solution. Measurement is done by a three-electrode-cell so that there is also a counter-electrode and a reference-electrode placed in the solution. The counter-electrode is also made of a Bucky-Paper so that 
the tested materials should have no electro-negativity. By charging the BPs an electrical field will be established like a capacitor which can be adjusted by the reference-electrode. The reference-electrode is a Kalomel-electrode which is arranged near by the analyzed Bucky-paper. The voltage is controlled by a potentiostat (Jaissle 1030 PC.T.) which is coupled to a function-generator (Yokogawa FG300). A data-acquisition system (Nicolet Technologies Vision) shows and saves the measured voltage, current and the deflection. Apart from this test the electro-mechanical system-response on dynamic incitation and a cyclovoltametry is done. This analysis-approach ${ }^{14}$ allows an intensive and consistent overview about the individual electro-mechanical BP-characteristics.

Because of its uneven build-up and possible bending-behavior because of ion-transfer ${ }^{19}$ the Nafionlayers are tested in an out-of-plane measuring facility that can be seen in figure 5 .
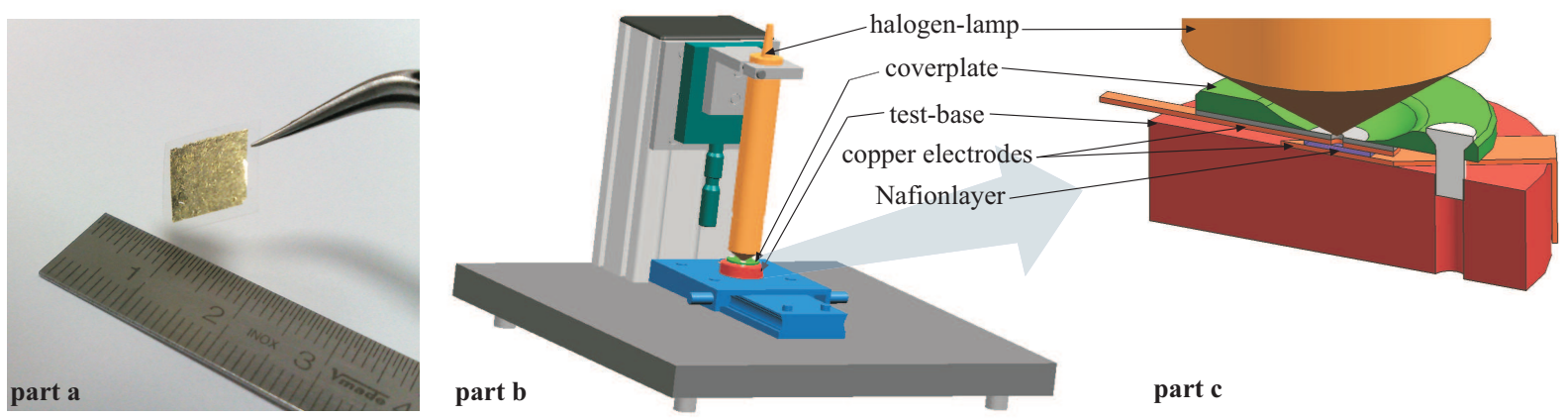

Figure 5. part a: Nafionsheet with three layers of nafion and gold foil, part b: test-rig, part c: detailed test-base with clamped Nafionlayer

This analysis takes place on a electrical-isolated synthetic base. On its top there are two copperelectrodes positioned with the tested Nafion-layer between. The top-electrode acting as counter-electrode has a small hole in its middle. The whole electrode-set-up is fixed by a coverplate with inserted plastic nuts. Through a hole in the coverplate and the top-electrode the halogen distance-device (Micro-Epsilon optoNCDT IFS 2400-0,08) can measure the changes directly on the top of the gold-coated Nafion-layer. The controlling data-storing is just the same like the first mentioned test-rig excepting the fact that there is no reference-electrode because of the small thickness of the Nafion-layers.

\section{EXPERIMENTAL RESULTS}

For the tests three different suppliers are dedicated for using their raw-material due to good conductibility a high purity of the SWCNT-material was decisive. Further Informations about the analyzed CNTproducts are available on the supplier-websites. ${ }^{20-22}$ All BPs are processed like it is described in chapter 3. The Elicarb-SWCNTs of Thomas Swan (T. Swan) which are produced by the common method of chemical-vapor-deposition (CVD) reach a purity of about 70\%. Six BPs of this Elicarb-SWCNTslot are analysed which performed by increasing the centrifuge-time (five, ten and fitfhteen minutes) with a better conductibility and Youngs's modulus (see table 1 and 2). There are two BPs made out of Unidym-material which is produced by a high-pressure carbon-monoxide chemical vapor deposition process (HiPCO). Throughout the process and afterward chemical cleaning a purity of about 95\% can be reached. Just one of the BPs was centrifuged which has also an enormous effect on the conductibility and stiffness. At least one BP was tested made of Nano-C material. This company developed a special pyrolyse-method $^{23}$ which guarantees with an additional chemical cleaning purities of $97 \%$, the highest avialable purity on the latest market. Referring to the raw-material condition it could be said that it is the more difficult to have a homogeneous CNT-dispersion the smaller the CNT-bulks of the powder are. It seems that fine powder therefore consists of very compact bulks which are difficult to break off by homogenizing. So it is not amazing that the material-loss after centrifuging can achieve $60 \%$ and more. So the process is not able to process all the raw-material on idealized way. An easy and stable processablilty of the raw-material can be seen by the number of the produced BPs, it is an inverse indicator for good usable material.

The table 1 also shows that not even the announced best purity guarantees an excellent conductibility which is decisive for a optimal actuator-performance. It is rather to discuss if chemical cleaning is an 


\begin{tabular}{|c|c|c|c|c|}
\hline supplier & product & manufacturing process & $\begin{array}{c}\text { purity } \\
{[\%]}\end{array}$ & $\begin{array}{c}\text { BP-conductibility } \\
{[\mathrm{S} / \mathrm{cm}]}\end{array}$ \\
\hline T. Swan & Elicarb SW & CVD & 70 & $62-241$ \\
\hline Unidym & super purified & HiPCO & 95 & $90-254$ \\
\hline Nano-C & nano-cpt & pyrolyse $^{23}$ & 97 & $46-81$ \\
\hline
\end{tabular}

Table 1. overview of CNT-material properties and suppliers

ideal process step for purifying the SWCNT-powder because it causes a downstream thermal reductive step to eliminate possible surface-modifications. Studies present that SWCNTs treated by these methods will never show similar conductibilities like untreated powder because of produced defects.

The produced Bucky-papers are analyzed by a scanning electron microscope (SEM, Leo 1350) with resolutions up to 200.000-times so even SWCNT-bundles are visible. Because of the filtration-process the top and bottom surfaces are very flat and uncover just a few details. Furthermore the contact with the membrane and the waterside-pressure may form the surface in a special compacted way so that just a cryogen broken paper shows its unaffected internal structure. Examples of those micrographs can be seen in figure 6 .
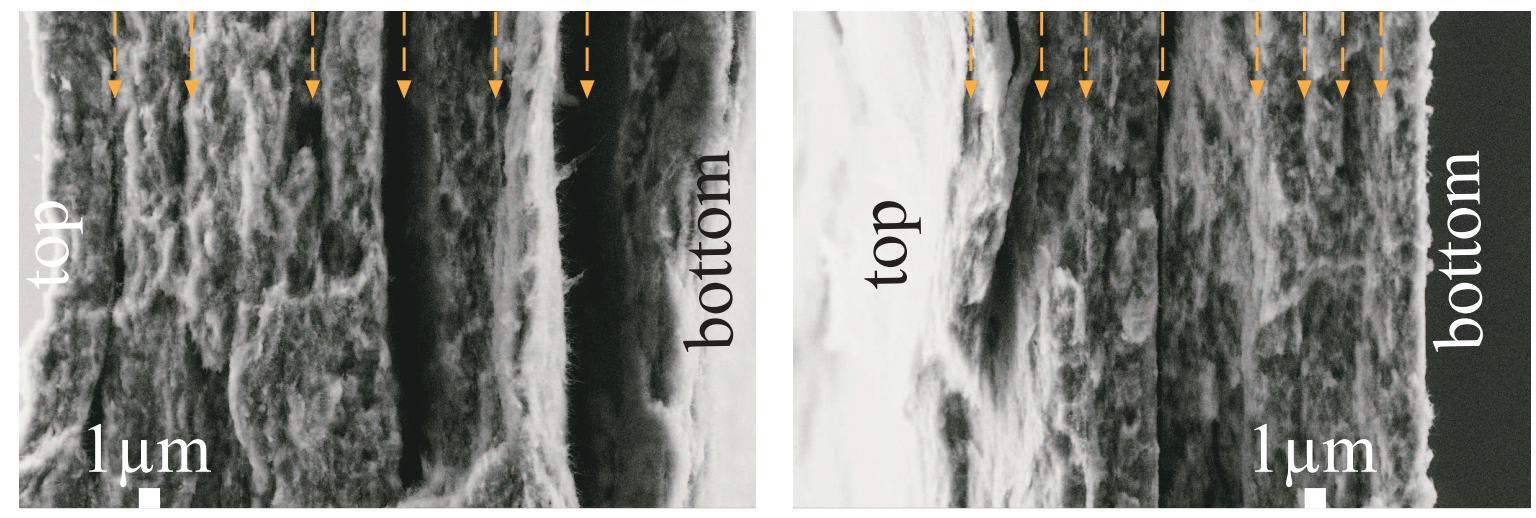

Figure 6. cryogen-cut through two different Bucky-papers presenting a layer-similar structure

The picture depicts a trend which is visible on many Bucky-papers not just on those made by highpressure filtration. Also vacuum-filtrated Bucky-papers seem to have a layer-like internal structure. Possibly the different fractions of dissolved particles lie down due to the gravity on the filtration-membrane building up this found structure. As well papers which were centrifuged show this build-up although they should consist of more homogeneous material. These pictures demonstrate that the random oriented Bucky-paper is more structural inhomogeneous than just a knotted cluster-like structure as it was considered to be before. A BP is rather build up of several layers (see orange arrows in fig. 6) of knotted CNT-cluster levels. With this fact it has to be discussed if a global Bucky-paper deflection can directly allow any statement about the reaction of the individual SWCNT-deflection.

The investigation on the deflection vs. specific Young's modulus of different Bucky-papers prove again an obvious influence of centrifuge-time on the active behavior of a Bucky-paper (see figure 7 and table 2)

There seems to be an analogue trend of increasing conductibility and Young's modulus. With increasing centrifuge-time the character of the individual SWCNT might dominate the global Bucky-paper instead of a bulk-character. Contrary to this the actuation decreases with bigger stiffness (see figure 7). Until now it is not possible to fabricate a randomly oriented BP with only the best characteristics. A higher stiffness has a significant impact on the actuation. With fig. 6 in mind the different characteristics are not that easy to compare. Another fact that is focused in fig. 7 is the consideration of the bulk-density of each BP which is calculated by the measured volume and weight. This data is respected using the specific Young's modulus as unity for the figure 7 .

The measurements made with different Nafion-layers (see table 3) demonstrate within the redox-band $( \pm 1 \mathrm{~V})$ no active reactions beside a distinctive drift. Because Nafion is a water-based material it should 


\begin{tabular}{|l|c|c|c|c|c|c|}
\hline supplier & $\begin{array}{c}\text { specimen- } \\
\mathrm{nr} .\end{array}$ & $\begin{array}{c}\text { BP-conductibility } \\
{[\mathrm{S} / \mathrm{cm}]}\end{array}$ & $\begin{array}{c}\text { centrifuge-time } \\
{[\mathrm{min}]}\end{array}$ & $\begin{array}{c}\text { bulk-density } \\
{\left[\mathrm{mg} / \mathrm{mm}^{3}\right]}\end{array}$ & $\begin{array}{c}\text { Young's modulus } \\
{[\mathrm{MPa}]}\end{array}$ & $\begin{array}{c}\text { deflection } \\
{[\%] \text { at } 0,7 \mathrm{~V}}\end{array}$ \\
\hline T. Swan & 1 & 62 & - & 0,592 & 732 & 0,12014 \\
\hline T. Swan & 2 & 115 & - & 0,729 & 979 & 0,12329 \\
\hline T. Swan & 3 & 151 & - & 0,638 & 1122 & 0,15752 \\
\hline T. Swan & 4 & 85 & - & 0,662 & 1142 & 0,06751 \\
\hline T. Swan & 5 & 219 & 10 & 0,72 & 2414 & 0,03082 \\
\hline T. Swan & 6 & 228 & 15 & 0,681 & 3424 & 0,02312 \\
\hline Unidym & 7 & 88 & - & 1,044 & 432 & 0,00984 \\
\hline Unidym & 8 & 128 & 5 & 0,774 & 1967 & 0,00732 \\
\hline Nano-C & 9 & 75 & - & 0,775 & 7385 & 0,00543 \\
\hline
\end{tabular}

Table 2. overview of analyzed SWCNT-Bucky-papers

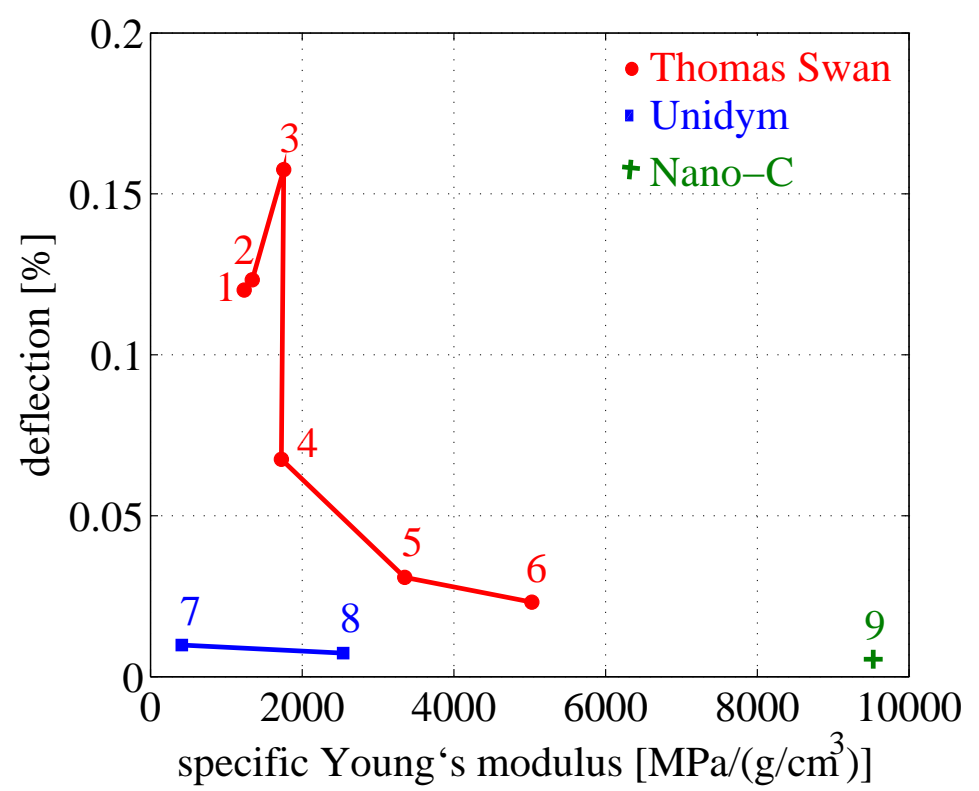

Figure 7. deflection and specific Young's modulus of three different Bucky-paper-lots

not be activated over $\pm 1 \mathrm{~V}$ but to investigate if Nafion has any response for higher voltages measurement were made at $\pm 3 \mathrm{~V}$. At least from $2 \mathrm{~V}$ up to $3 \mathrm{~V}$ there are some reactions, but because the tests are beyond the redox-band there will be oxidative or reductive chemical reactions of the components. The material and later on the actuator will be destroyed within an ongoing experiment within this voltages so that it is trivial for the actuator-application. A comparison of the weight before and after the tests (four hours long) shows a loss which is affected to the thermal activated vaporization of the contained water. A thermometry while testing offers a difference of just $1^{\circ} \mathrm{C}$.

\begin{tabular}{|c|c|c|}
\hline layers & $\begin{array}{c}\text { thickness } \\
{[\mu \mathrm{m}]}\end{array}$ & $\begin{array}{c}\text { weight loss after test } \\
{[\%]}\end{array}$ \\
\hline 2 & 49 & 0,43 \\
\hline 3 & 52 & 0,23 \\
\hline 4 & 70 & 0,15 \\
\hline \multicolumn{2}{|c|}{ Table 3. Overview of tested Nafion-layers }
\end{tabular}

These tests state if the actuator is activated within the redox-band, Nafion has no contribution to the deflection. Beside this fact it is not advisable to work beyond $\pm 1 \mathrm{~V}$ because Nafion will be corroded, toxic exhalation might appear and the actuator will not work in a stable mind for a longer time. 


\section{CONCLUSIONS}

The measurements and figures presented in this paper show that the passive constitution of the Buckypaper and its active response seems to be a very complex cross-linked system. Especially the dependencies between the BP-structure with its electro-mechanical characteristics and the active behavior of the SWCNT-mat are partially revealed but have to be worked on further. The structural dependence on the deflection is presented which seems to be a global effect beyond suppliers and purities. SEMmicrographs show that Bucky-papers can no longer be considered as a homogeneous framework. Due to its manufacturing-process a Bucky-paper is similar to several layers of cross linked depositions. Random oriented Bucky-papers are usable as model-material for handling with CNTs and for easy, cheap and fast CNT-mat manufacturing. With these results in mind it is proposed to use additional methods to investigate single CNTs and their individual deflection. At this point aligned structures such as arrays of aligned Bucky-papers have to be preferred.

In addition to the test on solid hybrid Nafion-CNT-actuators single Nafion-layers are investigated on their active response. It is described that within the only sensible voltage-band - the redox-band of water - are not any active strains measurable. So Nafion should be applicable for $\pm 1 \mathrm{~V}$ despite from its time-dependent reaction because of water-loss.

\section{ACKNOWLEDGMENTS}

This work was partly funded by the German Research Council (DFG) within the framework of the DFG PAK 355 - 'Basics for CNT-based Actuators' and the German Federal Ministry of Education and Research (BMBF) initiated project 'Aktu_Komp'.

\section{REFERENCES}

[1] H. P. Monner, S. Mühle, P. Wierach and J. Riemenschneider, "Carbon nanotubes - ein multifunktionaler Leichtbauwerkstoff für die Adaptronik," Adaptronic Congress (2003).

[2] R.H. Baughman, C. Cui, A. A. Zakhidov, Z. Iqbal, J. N. Barisci, G. M. Spinks, G. G. Wallace, A. Mazzoldi, D. D. Rossi, A. G. Rinzler, O. Jaschinski, S. Roth and M. Kerzesz,, "Carbon nanotube actuators," Science 284, 1340-1344 (1999).

[3] R. H. Baughman, "Materials synthesis: Towering forests of nanotubes trees," Nature Nano 1(2), 94-96 (2006).

[4] S. Ghosh, V. Gadagkar, A. K. Sood, "Strains induced in carbon nanotubes due to the precense of ions: Ab initio restricted hatree-fock calculations," Chemical Physics Letters (406), 10-14 (2005).

[5] B. J. Landi, R. P. Raffaelle, M. J. Heben, J. L. Alleman,W. VanDerveer and T. Gennett, "Single wall carbon nanotube-nafion composite actuators," Nano Letters 2(11), 1329-1332 (2002).

[6] B. J. Landi, R. P. Raffaelle, M. J. Heben, J. L. Alleman,W. VanDerveer and T. Gennett, "Development and characterisation of single wall carbon nanotube-nafion composite actuators," Material Science and Engineering B116(3), 359-362 (2005).

[7] I. A. Levitsky, P. Kanelos and W. B. Euler, "Electromechanical actuation of composite material from carbon nanotubes and ionomeric polymer," The Journal of Chemical Physics 121(2), 10581065 (2004).

[8] Suppiger, D., "Development of a Carbon Nanotube Actuator based on a solid Electrolyte," Dissertation, Centre of Structure Technologies ETHZ Zurich (2005).

[9] M. Shahinpoor and K. J. Kim, "Ionic polymer-metal composites: I. fundamentals," Smart Materials and Structures 10(4), 819-833 (2001).

[10] M. Shahinpoor and K. J. Kim, "Ionic polymer-metal composites: Iii. modeling and simulation as biomimetric sensor, actuators, transducers and artificial muscles," Smart Materials and Structures 13(6), 1362-1388 (2004).

[11] U. Kosidlo, D. G. Weis, K. Hying, M. H. Haque, I. Kolaric, "Development of Measurement Set-up for electromechanical Analysis of Bucky Paper Actuators," Journal of Nanotechnology Online 3, 1-11 (2007).

[12] B. J. Akle and D. J. Leo, "Characterization and modeling of extensional and bending actuation in ionomeric polymer transducers," Smart Materials and Structures 16, 1348-1360 (2007). 
[13] J. Riemenschneider, T. Mahrholz, H. P. Monner and J. Melcher, "System response of nanotube based actuators," Mechanics of Advanced Material and Structures 14(1), 57-65 (2007).

[14] J. Riemenschneider, H. Temmen and H. P. Monner, "CNT based actuators: Experimntal and theroretical inverstigation of the in-plain strain generation," Journal of Nanoscience and Nanotechnology 7(10), 3359-3364 (2007).

[15] A. Mazzoldi, D. D. Rossi and R. H. Baughman, "Electro-mechanical behavior of carbon nanotube sheets in electrochemical actuators," in SPIE, EAPAD, Y. Bar-Cohen, ed. , 3987 (2000).

[16] J. Riemenscheider, S. Opitz, M. Sinapius and H. P. Monner, "Modeling of carbon nanotube actuators: Part i - modeling and electrical properties," Journal of Intelligent Material Systems and Structures 20(3), 245-250 (2009).

[17] J. Riemenscheider, S. Opitz, M. Sinapius and H. P. Monner, "Modeling of carbon nanotube actuators: Part ii - mechanical properties, electro mechanical coupling and validation of the model," Journal of Intelligent Material Systems and Structures 20(3), 253-263 (2009).

[18] B. Vigolo, A. Pénicaud,C. Coulon, C. Sauder, R. Pailler, C. Journet, P. Bernier, P. Poulin, "Macroscopic Fibers and Ribbons of Oriented Carbon Nanotubes," Science 290, 1331-1334 (2000).

[19] P. G. de Gennes, K. Okumura, M. Shahinpoor, K. J. Kim, "Mechanoelectric effects in ionic gels," Europhysics Letters 50(4), 513-518 (2000).

[20] www.thomas.swan.co.uk (03.02.2010).

[21] www.unidym.com (03.02.2010).

[22] www.nano-c.com (03.02.2010).

[23] M. J. Height, J. B. Howard, J. W. Tester, J. B. Vander Sande, "Flame synthesis of single-walled carbon nanotubes," Carbon 42, 2295-2307 (2004). 